\title{
Lower-Bound Theorems for Pseudomanifolds
}

\author{
Tiong-Seng Tay
}

Department of Mathematics, National University of Singapore, 10 Kent Ridge Crescent, Singapore 0511 mattayts@nusunix.nus.sg

\begin{abstract}
We give purely combinatorial proofs of the lower-bound theorems for pseudomanifolds with or without boundary.
\end{abstract}

\section{Introduction}

Let $\Delta$ denote a simplicial complex and let $f_{k}(\Delta)$ denote the number of its $k$-dimensional faces. For positive integers $k, d, v, v_{\mathrm{i}}$, and $v_{\mathrm{b}}$ define the functions

$$
\begin{aligned}
& \varphi_{k}(v, d):= \begin{cases}v\left(\begin{array}{l}
d \\
k
\end{array}\right)-k\left(\begin{array}{l}
d+1 \\
k+1
\end{array}\right) & \text { if } \quad 1 \leq k \leq d-2, \\
(d-1) v-(d+1)(d-2) & \text { if } \quad k=d-1,\end{cases} \\
& \varphi_{k}^{b}\left(v_{\mathrm{i}}, v_{\mathrm{b}}, d\right):= \begin{cases}v_{\mathrm{b}}\left(\begin{array}{c}
d-1 \\
k
\end{array}\right)+v_{\mathrm{i}}\left(\begin{array}{l}
d \\
k
\end{array}\right)-k\left(\begin{array}{c}
d \\
k+1
\end{array}\right) & \text { if } \quad 1 \leq k \leq d-2, \\
v_{\mathrm{b}}+(d-1) v_{\mathrm{i}}-(d-1) & \text { if } \quad k=d-1 .\end{cases}
\end{aligned}
$$

The main aim of this paper is to prove the following lower-bound theorems. Throughout this paper $d$ is always an integer $\geq 3$ unless otherwise stated.

Theorem 1.1. Let $\Delta$ be $a(d-1)$-pseudomanifold with $v$ vertices. Then:

(i) $f_{k}(\Delta) \geq \varphi_{k}(v, d)$ if $1 \leq k \leq d-1$.

(ii) If equality holds for any $k, 1 \leq k \leq d-1$, then $\Delta$ is a stacked (d-1)-sphere for $d \geq 4$ and a triangulated 2-sphere for $d=3$.

Theorem 1.2. Let $\Delta$ be a $(d-1)$-pseudomanifold whose nonempty boundary is the disjoint union of normal pseudomanifolds. Suppose $\Delta$ has $v_{\mathrm{i}}$ vertices in the interior and 
$v_{\mathrm{b}}$ vertices in the boundary, then:

(i) $f_{k}(\Delta) \geq \varphi_{k}^{b}\left(v_{\mathrm{i}}, v_{\mathrm{b}}, d\right)$ if $1 \leq k \leq d-1$.

(ii) If $f_{k}(\Delta)=\varphi_{k}^{b}\left(v_{\mathrm{i}}, v_{\mathrm{b}}, d\right)$ for some $k, 1 \leq k \leq d-1$ then $\Delta$ is a stacked $(d-1)$-ball if $d \geq 4$ and a triangulated disk if $d=3$.

Kalai [8] proved these two theorems for manifolds and conjectured them to be true for pseudomanifolds and pseudomanifolds with arbitrary boundary (see also [1] and [2]). Previously, Klee [9] had proved the case $k=d-1$ of Theorem 1.1(i) while Björner [3] had conjectured Theorem 1.2(i). Kalai also pointed out, without giving details, that they would follow from the generic 3-rigidity of triangulated 2-manifolds. Later Fogelsanger [5] proved that triangulated 2-manifolds are generically 3-rigid. As a result of our investigation we think that Theorem 1.2 for the case of arbitrary boundary remains open.

In 1986 Gromov [7] defined a weaker form of rigidiry, $M_{d}$-rigidity, and proved that triangulated 2-manifolds are $M_{3}$-rigid. He then used this result to prove part (i) of Theorem 1.1. However, his proof of the $M_{3}$-rigidity of triangulated 2-manifolds had some gaps. These were subsequently corrected by Connelly and Whiteley [14]. We give a simple proof of this result. Then we use the techniques developed by Kalai [8] and the idea of $M_{d}$-rigidity to give a proof of Theorem 1.1. $M_{d}$-rigidity is a weaker form of generic rigidity as defined in [14]. The techniques used here have their roots in theory of generic rigidity.

Recently, Tay et al. [12] introduced the idea of skeletal rigidity of cell complexes, which generalizes infinitesimal rigidity of graphs. Connections between skeletal rigidity and the $g$-theorem of pl-spheres (piecewise linear spheres) have been made. It is conceivable that a weaker form of skeletal rigidity can be defined that generalizes $M_{d}$-rigidity. The techniques in this paper could then be generalized to yield a combinatorial proof of the $g$-theorem.

In writing up this paper we benefited greatly from the works of Kalai [8] and Gromov [7] and from continuing discussion with Walter Whiteley. For further historical note on the lower-bound theorems, readers are referred to [8].

The paper is organized as follows. In Section 2 we give the basic definitions and state the MPW (McMullen-Perles-Walkup) reduction which reduces the proof of the lower-bound theorem for certain simplicial complexes to the case $k=1$. In Section 3 we define normal pseudomanifolds and show that the lower-bound theorem for pseudomanifolds can be reduced to the case where the pseudomanifolds are normal. In Sections 4 and 5 we prove that triangulated 2 -manifolds are $M_{3}$-rigid and triangulated normal $(d-1)$-pseudomanifolds are $M_{d}$-rigid for $d \geq 4$. The proofs of Theorems 1.1 and 1.2 are presented in Section 5 and Section 6, respectively.

\section{Basic Definitions and MPW Reduction}

We use the following definitions and notations on simplicial complexes. Let $\Delta$ be a finite abstract simplicial complex on the vertex set $V$. Thus, $\Delta$ is a collection of subsets of $V$ (called the faces of $\Delta$ ) and if $\tau \in \Delta$ and $\sigma \subseteq \tau$, then $\sigma \in \Delta$. For $\sigma \in \Delta$ 
the dimension of $\sigma$ is $\operatorname{dim} \sigma:=|\sigma|-1 . f_{k}(\Delta)$ denotes the number of $k$-dimensional faces (or briefly $k$-faces) of $\Delta . V(\Delta)$ is the set of vertices (0-faces) of $\Delta$. 1-faces of $\Delta$ are called edges. The dimension of $\Delta$ is the maximum dimension of its faces. A one-dimensional simplicial complex is called a graph. The graph $G(\Delta)$ of $\Delta$ is the graph obtained by taking the vertices and edges of $\Delta$. For a face $\sigma \in \Delta$ the link of $\sigma$ in $\Delta$ is $\operatorname{lk}(\sigma, \Delta):=\{\tau-\sigma: \tau \in \Delta, \tau \supseteq \sigma\}$.

A simplicial complex $\Delta$ is pure if all its maximal faces have the same dimension. Maximal faces of a pure simplicial complex are called facets. Two facets $\sigma, \tau$ of a pure simplicial complex are adjacent if they intersect in a maximal proper face of each. A pure simplicial complex $\Delta$ is strongly connected if, for every two facets $\sigma$ and $\tau$ of $\Delta$, there is a sequence of facets $\sigma=\sigma_{0}, \sigma_{1}, \ldots, \sigma_{m}=\tau$, such that $\sigma_{i}$ and $\sigma_{i+1}$ are adjacent of $0 \leq i<m . \Delta$ is said to be connected if its graph is strongly connected. It follows from the definition that strong connectedness and connectedness are equivalent for a one-dimensional simplicial complex.

Every simplicial complex $\Delta$ is associated with a topological space, denoted by $|\Delta|$ (see [10]). A d-pseudomanifold is a strongly connected $d$-dimensional simplicial complex such that every $(d-1)$-face is contained in exactly two facets. A $d$-pseudomanifold $\Delta$ is a triangulated $d$-manifold if $|\Delta|$ is a manifold.

Remark 2.1. By a process known as MPW reduction, if a class of simplicial complex is closed under taking links, then the lower-bound theorem for this class follows from the case $k=1$. (See [8] for details.)

\section{Pseudomanifolds Without Boundary}

The class of pseudomanifolds is not closed under taking links. The pinched torus is an example (Fig. 3.1). Thus the MPW reduction does not apply directly. Therefore we go to the class of normal pseudomanifolds: a $(d-1)$-pseudomanifold is normal if every $k$-face, $0 \leq k<d-2$, has a connected link. Note that, by Proposition 3.1 below, $\operatorname{lk}(\sigma, \Delta)$, for every $k$-face $\sigma, 0 \leq k<d-2$, is strongly connected. Thus the class of normal pseudomanifolds is closed under taking links of faces of dimension $<d-2$, whence part (i) of the theorem for a normal $(d-1)$-pseudomanifold reduces by MPW reduction to the case $k=1$.
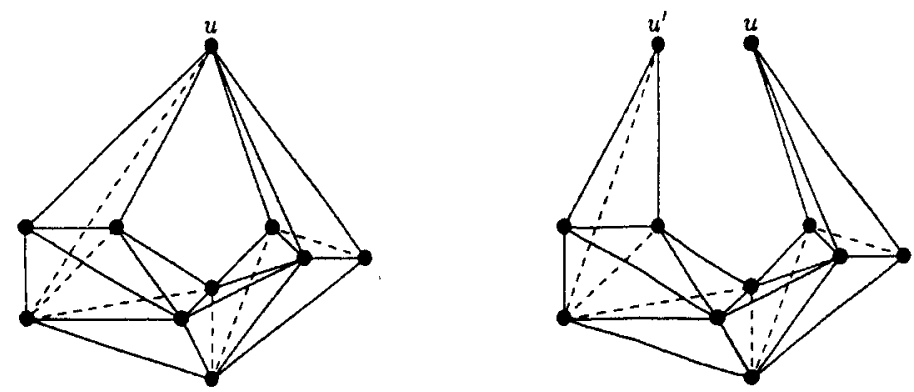

Fig. 3.1. Normalization of the "pinched" torus: The pinched torus (left) is a pseudomanofold. "Pulling apart" at $u$ yields a normal pseudomanofold. 
Proposition 3.1. Let $\sigma$ be a $k$-face of a normal ( $d-1)$-pseudomanifold, $0 \leq k<d$ -2 . Then $\operatorname{lk}(\sigma, \Delta)$ is strongly connected.

Proof. If $k=d-3$, then $\operatorname{lk}(\sigma, \Delta)$ is a one-dimensional simpiicial complex. In this case connectedness and strong connectedness are equivalent.

Suppose $k<d-3$ and $\operatorname{lk}(\sigma, \Delta)$ is not strongly connected. Partition its facets into strongly connected subcomplexes, all of dimension $d-k-2$. Since $\operatorname{lk}(\sigma, \Delta)$ is connected, two of these subcomplexes, say $\Delta_{1}$ and $\Delta_{2}$, have some vertices in common. Let $\tau$ be a maximal face in $\Delta_{1} \cap \Delta_{2}$. Then $\operatorname{dim}(\tau) \leq d-k-4$ and $\operatorname{dim}(\sigma \cup \tau)<d-2$. However, $\operatorname{lk}(\sigma \cup \tau, \Delta)=\operatorname{lk}(\tau, \operatorname{lk}(\sigma, \Delta))$ and this is not connected. Thus we have a contradiction.

We can turn an arbitrary pseudomanifold into a normal one by "pulling apart" at certain faces. (See Fig. 3.1 for an example.) This normalization process is described on p. 83 of [11]. (See also p. 151 of [6] and [8].)

As a consequence, for part (i) of Theorem 1.1 it suffices to prove the following:

Theorem 3.2. Let $\Delta$ be a normal $(d-1)$-pseudomanifold with v vertices. Then

$$
f_{1}(\Delta) \geq \varphi_{1}(v, d)=d v-\left(\begin{array}{c}
d+1 \\
2
\end{array}\right) .
$$

We devote the next two sections to proving this result as well as part (ii) of Theorem 1.1.

\section{Triangulated 2-Manifolds are $M_{3}$-Rigid}

Given a graph, its subgraphs are its subcomplexes. A subgraph is spanning if it includes all the vertices of the graph. For a simplicial complex we say that a face $\sigma$ meets a given set $U$ of vertices of $\sigma \cap U \neq \emptyset$.

Definition 4.1. A graph $G=(V, E)$ is $M_{3}$-rigid if, for every triple of vertices $a, b$, and $c$ and for every $V^{*} \subseteq V-\{a, b, c\}$, we have $\left|E^{*}\right| \geq 3\left|V^{*}\right|$, where $E^{*}$ is the set of all edges that meet $V^{*}$.

Clearly, a graph is $M_{3}$-rigid if and only if it contains a spanning subgraph which is $M_{3}$-rigid.

As an immediate consequence of the definition we have

Proposition 4.2. Suppose a graph $G=(V, E)$ contains a triangle and is $M_{3}$-rigid, then

$$
|E| \geq 3|V|-6 \text {. }
$$

However, the $M_{3}$-rigidity of $G=(V, E)$ does not imply that $|E| \geq 3|V|-6$ if $G$ does not have a triangle. In particular, the graph obtained from the complete bipartite graph $K_{6,6}$ by deleting six edges symmetrically and one other edge is $M_{3}$-rigid by the following proposition. 
Proposition 4.3. Suppose $G=(V, E)$ is a graph with $|E|=3|V|-t$, where $t$ is an integer. Then $G$ is $M_{3}$-rigid if and only if every subgraph $H=\left(V^{\prime}, E^{\prime}\right)$ with at least three vertices satisfies

$$
\left|E^{\prime}\right| \leq 3\left|V^{\prime}\right|-t
$$

Proof. Let $H=\left(V^{\prime}, E^{\prime}\right)$ be any subgraph with at least three vertices. Let $V^{*}=V$ $-V^{\prime}$. Then $\left|E^{*}\right| \geq 3\left|V^{*}\right|$ if and only if

$$
\left|E^{\prime}\right|=|E|-\left|E^{*}\right| \leq|E|-3\left|V^{*}\right|=3|V|-t-3\left|V^{*}\right|=3\left|V^{\prime}\right|-t .
$$

The graphical interpretation of $M_{3}$-rigidity is the following.

Proposition 4.4. A graph $G=(V, E)$ is $M_{3}$-rigid if and only if, for every triple of vertices $a, b, c$, there are three edge-disjoint matchings of $V-\{a, b, c\}$ with adjacent edges.

Proof. We need to use Hall's theorem on matchings in bipartite graphs. Define a bipartite graph whose bipartitions are $A:=V-\{a, b, c\}$ and $B:=E$ with the obvious adjacency. Then the result is just an easy consequence of Hall's theorem.

Suppose we identify the three vertices $a, b$, and $c$ and call the resulting graph $G_{a b c}$. Then each of the matchings described in Proposition 4.4 corresponds to a spanning subgraph of $G_{a b c}$ with $\left|V\left(G_{a b c}\right)\right|-1$ edges. This subgraph is a spanning tree if it is connected; otherwise it has two components. This subgraph may not be a spanning tree as shown by the following example.

Example 4.5. Let $G$ be the graph obtained by joining two disjoint copies of $K_{7}$, the complete graph on seven vertices, using two edges. It can be shown (by using Proposition 4.3 with $t=-2$ ) that $G$ is $M_{3}$-rigid. Since $G$ is two-edge connected, $G_{a b c}$ does ot contain three edge-disjoint spanning trees.

Remark 4.6. The term $M_{3}$-rigidity was coined by Walter Whiteley. Gromov used the term 3-rigidity which has a different meaning in rigidity theory. Originally Gromov defined $M_{d}$-rigidity for two-dimensional simplicial complexes and the three vertices $\{a, b, c\}$ were required to form a face of the complex. However, with the more general definition, we can still prove $M_{3}$-rigidity of triangulated 2-manifolds.

Remark 4.7. Connections with rigidity: We now say something about generic rigidity which has been mentioned earlier. A bar framework in $d$-space consists of a set of rigid bars which are linked together at their ends using ball joints. It is infinitesimally rigid if no infinitesimal deformation of the vertices is possible without infinitesimally altering the edge lengths. Such a framework has an underlying graph. A graph is said to be generically $d$-rigid if it is the underlying graph of some infinitesimally rigid bar framework in $d$-space. (See [14].)

Unlike generic rigidity, $M_{3}$-rigidity does not give rise to a matroid because minimally $M_{3}$-rigid spanning subgraphs of a complete graph can have different 
numbers of edges (Propositions 4.3 and 4.2). (See [14] for a discussion on matroids and rigidity.)

We say that a graph $G=(V, E)$ satisfies the $d$-count if every subgraph $H=$ $\left(V^{\prime}, E^{\prime}\right)$ with $\left|V^{\prime}\right| \geq d$ satisfies

$$
\left|E^{\prime}\right| \leq d\left|V^{\prime}\right|-\left(\begin{array}{c}
d+1 \\
2
\end{array}\right)
$$

with equality when $H=G$. (In the literature on rigidity, this condition is also known as Laman's condition or Maxwell's condition.)

If the bar framework in $d$-space on graph $G$ is minimally generically $d$-rigid, then it satisfies the $d$-count. However, the converse is true only when $d=2$. (This is known as Laman's theorem, see [14].) Later (Section 7) we give an example to show that $M_{3}$-rigidity is weaker than generic 3-rigidity.

To prove that the graph of every triangulated 2-manifold is $M_{3}$-rigid, we need a result from topological graph theory. (See Sections 6.2 and 11.1 of [13]) A graph is embedded in a surface $M$ if it is drawn in $M$ so that edges intersect only at their common vertices. Such an embedding partitions $M$ into regions which are the components of the 2-manifold after the edges are deleted.

For a manifold $M$ we denote its genus by $g(M)$ if it is oriented and by $\vec{g}(M)$ if it is nonoriented. We denote the oriented and nonoriented genus of a graph $G$ by $g(G)$ and $\tilde{g}(G)$, respectively. The next series of lemmas can be found in [13]. A graph $G$ has $e$ edges and $v$ vertices and the corresponding numbers for its subgraph $G_{i}$ are $e_{i}$ and $v_{i}$.

Lemma 4.8. If a graph $G$ is connected with $v \geq 3$, then

$$
e \leq\left\{\begin{array}{l}
3 v+6 g(G)-6, \\
3 v+3 \tilde{g}(G)-6 .
\end{array}\right.
$$

Lemma 4.9. For any graph $G, \tilde{g}(G) \leq 2 g(G)+1$.

Lemma 4.10. If $H$ is a subgraph of $G$, then $g(H) \leq g(G)$ and $\tilde{g}(H) \leq \tilde{g}(G)$.

Lemma 4.11. Let $\mu(G):=\max \{2-2 g(G), 2-\tilde{g}(G)\}$, then $\mu(G)=2-\tilde{g}(G)$ or $3-\tilde{g}(G)$.

Proof. If $\mu(G) \neq 2-\tilde{g}(G)$, then $\tilde{g}(G)>2 g(G)$. Hence $\tilde{g}(G)=2 g(G)+1$ and the result follows.

Lemma 4.12. If $G_{1}, \ldots, G_{k}$ are the connected components of a graph $G$, then

$$
g(G)=\sum g\left(G_{i}\right)
$$

and

$$
\tilde{g}(G)= \begin{cases}1+k+\sum \tilde{g}\left(G_{i}\right) & \text { if } \tilde{g}(G)=2 g(G)+1 \\ 2 k-\sum \mu\left(G_{i}\right) & \text { otherwise. }\end{cases}
$$


Proposition 4.13. Suppose $G$ is a graph with $v(\geq 3)$ vertices and e edges. If $G$ is embedded in a 2-manifold with Euler characteristic $\chi$, then

$$
e \leq 3 v-3 \chi .
$$

Equality holds if and only if $G$ is connected and all the regions are triangles.

Proof. First note that $\chi=2-2 g(M)$ if $M$ is oriented and $\chi=2-\tilde{g}(M)$ if $M$ is not oriented. If $G$ is connected, then the result follows from Lemma 4.8.

So we assume that $G_{1}, \ldots, G_{k}$ are the connected components of $G$. From Lemmas 4.8 and 4.12 , we have

$$
\begin{aligned}
e & \leq 3 v+6 \sum g\left(G_{i}\right)-6 k \\
& \leq 3 v+6 g(G)-6 .
\end{aligned}
$$

If $\tilde{g}(G)=2 g(G)+1$, then $e \leq 3 v+3 \tilde{g}(G)-9 \leq 3 v+3 \tilde{g}(G)-6$.

In the remaining case $\tilde{g}(G) \leq 2 g(G)$. Since $\mu\left(G_{i}\right) \leq 3-\tilde{g}\left(G_{i}\right)$, from Lemma 4.12,

$$
\begin{aligned}
e & \leq 3 v+3 \sum \tilde{g}\left(G_{i}\right)-6 k \\
& \leq 3 v-3 \sum \mu\left(G_{i}\right)+3 k \\
& \leq 3 v+3 \tilde{g}(G)-6 k+3 k \\
& \leq 3 v+3 \tilde{g}(G)-6 .
\end{aligned}
$$

The last inequality follow because $k \geq 2$.

We are now ready to prove the main theorem of this section.

Theorem 4.14. The graph of every triangulated 2-manifold without boundary is $M_{3}$-rigid.

Proof. If the graph of the 2-manifold $\Delta$ has $v$ vertices, $e$ edges, and the Euler

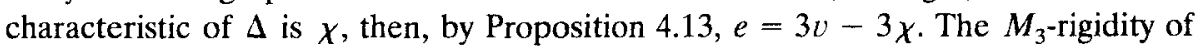
$\Delta$ then follows from Propositions 4.3 and 4.13 .

\section{5. (d-1)-Pseudomanifolds are $M_{d}$-Rigid}

Definition 5.1. A simplicial $(d-1)$-complex, $d \geq 4, \Delta$ is $M_{d}$-rigid if, for every $(d-1)$-simplex $\sigma$ and for every $V^{*} \subseteq \mathrm{V}(\Delta)-\sigma$, we have $\left|E^{*}\right| \geq d\left|V^{*}\right|$, where $E^{*}$ is the set of all edges that meet $V^{*}$.

The following is an immediate consequence of the definition.

Proposition 5.2. Suppose $\Delta$ is a simplicial $(d-1)$-complex which is $M_{d}$-rigid. Then

$$
f_{1}(\Delta) \geq d f_{0}(\Delta)-\left(\begin{array}{c}
d+1 \\
2
\end{array}\right)
$$




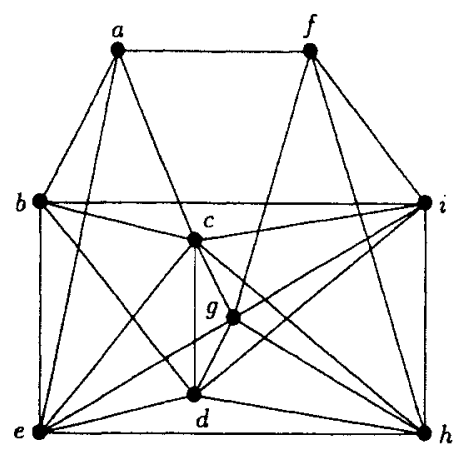

Fig. 5.1. Graph $G$.

Unlike $M_{3}$-rigidity, $M_{d}$-rigidity for $d \geq 4$ is a property of $\Delta$ rather than its graph (Example 5.3). However, if the graph of a simplicial $(d-1)$-complex $\Delta$ contains a spanning subgraph which satisfies the $d$-count, then $\Delta$ is $M_{d}$-rigid (see Proposition 5.4 below with

$$
t=\left(\begin{array}{c}
d+1 \\
2
\end{array}\right)
$$

Thus $\Delta$ is $M_{d}$-rigid if its graph is generically $d$-rigid.

Example 5.3. Consider the graph $G$ shown in Fig. 5.1. Let $\Delta$ be the simplicial 3-complex with $\{b, c, d, e\}$ and the other edges of $G$ as its maximal cells. Let $L$ be the simplicial 3-complex with $\{a, b, c, e\}$ and the other edges of $G$ as its maximal cells. Then $\Delta$ is not $M_{4}$-rigid for if $V^{*}=\{a, f\}$, then $\left|E^{*}\right|<4\left|V^{*}\right|$. By checking all possibilities it can be shown that $L$ is $M_{4}$-rigid. However, both have the same graph.

Proposition 5.4. Suppose $\Delta$ is a simplicial (d-1)-complex satisfying $f_{1}(\Delta)=$ $d f_{0}(\Delta)-t$ for some integer $t$. Then $\Delta$ is $M_{d}$-rigid if and only if, for every subgraph $H$ of $\Delta^{\prime}$ which contains the vertices of $a(d-1)$-simplex, $|E(H)| \leq d|V(H)|-t$.

This is a direct generalization of Proposition 4.3 with similar proof. Thus we omit the proof. The following is an immediate consequence.

Corollary 5.5. A simplicial $(d-1)$-complex $\Delta$ is $M_{d}$-rigid if its graph contains a spanning subgraph which satisfies the d-count.

Let $\Delta$ be a simplicial $(d-1)$-complex and let $u$ be a new vertex. The one-point cone with $u$ is the simplicial $d$-complex

$$
\Delta * u=\{\sigma \cup\{u\}: \sigma \in \Delta\} \cup \Delta .
$$

The following two results are crucial to the proof of the main theorem in this section. They are analogues of similar theorems in rigidity theory. These two results and Theorem 5.9 are known to Gromov who gave a sketch of their proof in [7]. 
Proposition 5.6. If a simplicial $(d-1)$-complex $\Delta$ is $M_{d}$-rigid, then the one-point cone with a new vertex $u$ is $M_{d+1}$-rigid.

Proof. Take any $d$-simplex $\sigma$ in $\Delta * u$. This simplex contains $u$, whence $\sigma-\{u\}$ is a $(d-1)$-simplex in $\Delta$. Let $V^{*} \subseteq V(\Delta * u)-\sigma$. Then $V^{*} \subseteq V(\Delta)-(\sigma-\{u\})$. Thus $E^{\prime}$, the set of all edges in $\Delta$ that meet $V^{*}$, satisfies $\left|E^{\prime}\right| \geq d\left|V^{*}\right|$. If $E^{*}$ is the set of all edges in $\Delta * u$ that meet $V^{*}$, then

$$
\left|E^{*}\right|=\left|E^{\prime}\right|+\left|V^{*}\right| \geq(d+1)\left|V^{*}\right| \text {. }
$$

Proposition 5.7. Suppose $\Delta_{1}$ and $\Delta_{2}$ are two $M_{d}$-rigid simplicial $(d-1)$-complexes and $\Delta_{1} \cap \Delta_{2}$ contains $a(d-1)$-simplex. Then $\Delta_{1} \cup \Delta_{2}$ is $M_{d}$-rigid.

Pronf. Take any $(d-1)$-simplex $\sigma$ in $\Delta_{1} \cup \Delta_{2}$. Assume for convenience that $\sigma$ is in $\Delta_{1}$. Let $U^{*} \subseteq V\left(\Delta_{1} \cup \Delta_{2}\right)-\sigma, U_{1}=U^{*} \cap V\left(\Delta_{1}\right)$, and $U_{2}=U^{*}-U_{1}$. Suppose $E_{1}$ is the set of all edges in $\Delta_{1}$ that meet $U_{1}$, and $E_{2}$ is the set of all edges in $\Delta_{2}-\Delta_{1}$ that meet $U_{2}$. Then $E^{*}$, the set of all edges of $\Delta_{1} \cup \Delta_{2}$ that meet $U^{*}$, contains $E_{1} \cup E_{2}$. By the $M_{d}$-rigidity of $\Delta_{1}$, we have $\left|E_{1}\right| \geq d\left|U_{1}\right|$. Also, since $U_{2} \subseteq V\left(\Delta_{2}\right)-V\left(\Delta_{1} \cap \Delta_{2}\right)$ and $\Delta_{1} \cap \Delta_{2}$ contains a $(d-1)$-simplex, we have, by the $M_{d}$-rigidity of $\Delta_{2},\left|E_{2}\right| d\left|U_{2}\right|$. Thus

$$
\left|E^{*}\right| \geq\left|E_{1}\right|+\left|E_{2}\right| \geq d\left|U_{1}\right|+d\left|U_{2}\right|+d\left|U^{*}\right| .
$$

Remark 5.8. Our definition of $M_{d}$-rigidity for $d>3$ coincides with that of Gromov. The main reason that we cannot relax the condition to all arbitrary sets of $d$ vertices is that Proposition 5.7 will no longer be true.

With these we can now prove that all normal $(d-1)$-pseudomanifolds are $M_{d}$-rigid.

Theorem 5.9. All normal $(d-1)$-pseudomanifolds are $M_{d}$-rigid.

Proof. We prove this by induction on $d$. Since the normal 2-pseudomanifolds are just triangulated 2-manifolds, the theorem is true for $d=3$.

Assume that the result is true for $d-1$. Let $\Delta$ be a normal $d$-pseudomanifold and let $\mu$ be one of its vertices. Then $\operatorname{lk}(\mu, \Delta)$ is a normal $(d-1)$-pseudomanifold. By the induction hypothesis, $\operatorname{lk}(\mu, \Delta)$ is $M_{d}$-rigid. Therefore the cone $\operatorname{lk}(\mu, \Delta) * u$ is $M_{d+1}$-rigid. If $w$ is a vertex adjacent to $u$, then the cones of their $\operatorname{links,} \operatorname{lk}(\mu, \Delta) * u$ and $\operatorname{lk}(w, \Delta) * w$, have a $d$-simplex in common, whence their union is $M_{d+1}$-rigid. Repeated applications show that $\Delta$ is $M_{d+1}$-rigid.

We can now prove Theorem 1.1.

Proof of Theorem 1.1. Part (i) is an immediate consequence of Theorem 5.9 and the MPW reduction.

For part (ii), if equality holds for some $k$, then by the normalization procedure in Section $3, \Delta$ is a normal pseudomanifold. For $d=3$, a normal 2-pseudomanifold $\Delta$ 
is just a triangulated 2-manifold. So $f_{i}(\Delta)=\varphi_{i}(v, 3)$ for $i=1$ or 2 implies that $\Delta$ is a triangulated sphere.

For $d>3, f_{k}(\Delta)=\varphi_{k}(v, d)$ for some $k, 1 \leq k<d$, implies by the MPW reduction that

$$
f_{1}(\Delta)=\varphi_{1}(v, d)=d v-\left(\begin{array}{c}
d+1 \\
2
\end{array}\right)
$$

For all $u \in V(\Delta), \operatorname{lk}(u, \Delta)$, being a normal ( $d-2)$-pseudomanifold, is $M_{d-1}$-rigid. Thus the cone $\mathrm{lk}(u, \Delta) * u$ is $M_{d}$-rigid, whence

$$
e^{*} \geq d v^{*}-\left(\begin{array}{c}
d+1 \\
2
\end{array}\right)
$$

where $e^{*}$ and $v^{*}$ are the number of vertices and edges of the cone respectively. By Proposition 5.4 with

$$
t=\left(\begin{array}{c}
d+1 \\
2
\end{array}\right) \text {, we have } e^{*} \leq d v^{*}-\left(\begin{array}{c}
d+1 \\
2
\end{array}\right) \text {, whence } e^{*}=d v^{*}-\left(\begin{array}{c}
d+1 \\
2
\end{array}\right)
$$

By the construction of the cone, $\operatorname{lk}(u, \Delta)$ satisfies $f_{1}(\operatorname{lk}(u, \Delta))=\varphi_{1}\left(v^{*}-1, d-1\right)$. Thus $\mathrm{k}(u, \Delta)$ is a triangulated $(d-2)$-sphere. Since this is true for every vertex $u$, $\Delta$ is a triangulated $(d-1)$-manifold. Kalai [8] has shown that a triangulated $(d-1)$-manifold $\Delta$ which satisfies

$$
f_{1}(\Delta)=f_{0}(\Delta)-\left(\begin{array}{c}
d+1 \\
2
\end{array}\right)
$$

is a stacked sphere, thus completing the proof.

\section{Manifolds and Pseudomanifolds with Boundary}

A $d$-pseudomanifold with boundary $\Delta$ is a strongly connected $d$-dimensional simplicial complex such that every $(d-1)$-face is contained in at most two facets. The boundary of $\Delta, \partial \Delta$, is the $(d-1)$-dimensional pure simplicial complex whose facets are those $(d-1)$-faces of $\Delta$ which are included in a unique facet of $\Delta . \Delta$ is a triangulated $d$-manifold with boundary if $|\Delta|$ is a $d$-manifold with boundary.

Let $\Delta$ be a simplicial complex and let $\sigma$ be a face of $\Delta$. The antistar of $\sigma$ is defined by $\operatorname{ast}(\sigma, \Delta):=\{\tau \in \Delta: \tau \cap \sigma=\emptyset\}$. A simplicial complex is a stacked $(d-1)$-ball if it is the antistar of a vertex of a stacked $(d-1)$-sphere.

By the definition a stacked $(d-1)$-ball $\Theta$ is a triangulated $(d-1)$-manifold with boundary. If $\Theta=\operatorname{ast}(u, \Omega)$, where $\Omega$ is a stacked $(d-1)$-sphere and $u$ is a vertex of $\Omega$, then the facets of the boundary of $\Theta$ are the $(d-2)$-faces of $1 \mathrm{k}(u, \Omega)$. Suppose $\Theta$ has $v$ vertices, with $v_{\mathrm{b}}$ of them on the boundary and $v_{\mathrm{i}}$ in the interior. Then $v_{\mathrm{b}} \geq d$. Since $f_{k}(\Theta)=f_{k}(\Omega)-f_{k-1}(1 \mathrm{k}(u, \Omega))$, direct computation shows that 
$f_{k}(\Theta)=\varphi_{k}^{b}\left(v_{\mathrm{i}}, v_{\mathrm{b}}, d\right)$. Thus Theorem 1.2 is true for stacked balls. To prove Theorem 1.2 in general we need the following preliminary results. The proofs of these are similar to the analogous results in [8] and are thus omitted.

Proposition 6.1. Let $\Delta$ be a $(d-1)$-dimensional pure simplicial complex which is $M_{d}$-rigid. For any vertex $u$ of $\Delta, \gamma(\operatorname{lk}(u, \Delta)) \leq \gamma(\Delta)$.

Proposition 6.2. Let $\Delta$ be an $M_{d^{-}}$rigid $(d-1)$-pseudomanifold and let $u$ be one of its vertices. Suppose the link of every face of $\Delta$ is connected, except possibly $1 \mathrm{k}(u, \Delta)$. Then

$$
\gamma_{k}(\Delta) \geq \gamma_{k-1}(\mathrm{lk}(u, \Delta)), \quad 1 \leq k \leq d-2 .
$$

If equality holds, then $\gamma(\Delta)=0$.

We are now ready to prove Theorem 1.2 .

Proof of Theorem 1.2. The case $d=3$ is quite simple. Note that normalization increases either $v_{\mathrm{i}}$ or $v_{\mathrm{b}}$, and therefore increases $\varphi_{k}^{b}\left(v_{\mathrm{i}}, v_{\mathrm{b}}, 3\right)$ while keeping $f_{k}(\Delta)$ unchanged. Thus we need only consider that normal 2-pseudomanifolds with boundary are just triangulated 2-manifolds with boundary. For a triangulated 2-manifold $\Delta$ with Euler characteristic $\chi$ and $t$ connected components in the boundary, we have

$$
f_{1}(\Delta)=3 v_{\mathrm{i}}+2 v_{\mathrm{b}}+3 t-3 \chi \geq 3 v_{\mathrm{i}}+2 v_{\mathrm{b}}-3
$$

and

$$
f_{2}(\Delta)=2 v_{\mathrm{i}}+v_{\mathrm{b}}+2 t-2 \chi \geq 2 v_{\mathrm{i}}+v_{\mathrm{b}}-2
$$

with equality if and only if $\chi=2$ and $t=1$, i.e., $\Delta$ is a 2-ball.

For $d \geq 4$, let $u$ be a vertex not in $\Delta$ and let $\Delta^{\prime}$ be the complex $\Delta \cup(\partial \Delta * u)$. By the given conditions on the links of $\Delta$, for each vertex $x$ different from $u, \operatorname{lk}\left(x, \Delta^{\prime}\right)$ is a normal $(d-2)$-pseudomanifold and is hence $M_{d-1}$-rigid. It is an easy consequence of Propositions 5.6 and 5.7 that $\Delta^{\prime}$ is $M_{d}$-rigid.

Also note that $f_{0}\left(\Delta^{\prime}\right)=v_{i}+v_{b}+1$. Recall that, for $j \geq 1, \gamma_{j}\left(\Delta^{\prime}\right)=f_{j}\left(\Delta^{\prime}\right)-$ $\varphi_{j}(n, d)$. Put $\gamma_{0}\left(\Delta^{\prime}\right)=0$. A simple inspection shows that

$$
f_{j}(\Delta)-\varphi_{j}^{b}\left(v_{\mathrm{i}}, v_{\mathrm{b}}, d\right)=\gamma_{j}\left(\Delta^{\prime}\right)-\gamma_{j-1}\left(\mathrm{lk}\left(u, \Delta^{\prime}\right)\right)
$$

We apply the normalization procedure to $\Delta^{\prime}$. Let $\sigma \neq\{u\}$ be a face of minimum dimension $k, k<d-2$, such that $\operatorname{lk}\left(\sigma, \Delta^{\prime}\right)$ has $t$ connected components. (Note that by the condition on the boundary, $u \notin \sigma$.) We pull apart $\Delta^{\prime}$ at $\sigma$ to obtain $N_{\sigma}\left(\Delta^{\prime}\right)$ and wish to show that

$$
\left\{\gamma_{j}\left(\Delta^{\prime}\right)-\gamma_{j}\left(N_{\sigma}\left(\Delta^{\prime}\right)\right)\right\}+\left\{\gamma_{j-1}\left(\operatorname{lk}\left(u, N_{\sigma}\left(\Delta^{\prime}\right)\right)\right)-\gamma_{j-1}\left(\operatorname{lk}\left(u, \Delta^{\prime}\right)\right)\right\} \geq 0
$$


Since $N_{\sigma}\left(\Delta^{\prime}\right)$ is obtained by replacing $\sigma$ with $t$ copies of it, $\sigma_{1}, \sigma_{2}, \ldots, \sigma_{t}$; and replacing each face $\sigma \cup \tau$ with $\sigma_{i} \cup \tau$ if $\tau$ is in the $i$ th component of $\operatorname{lk}\left(\sigma, \Delta^{\prime}\right)$, the first difference is always positive. Since $u \notin \sigma$, the second difference is zero because pulling apart at $\sigma$ does not affect $\operatorname{lk}\left(u, \Delta^{\prime}\right)$. Thus inequality (6.1) follows.

Hence we can assume that $\Delta^{\prime}$ satisfies the conditions of Proposition 6.2. From this part (i) follows

To prove part (ii) we first note that equality implies $\gamma\left(\Delta^{\prime}\right)=0$. By Theorem 1.1, $\Delta^{\prime}$ is a stacked $(d-1)$-sphere. Hence $\Delta$ is a stacked $(d-1)$-ball.

\section{Concluding Remarks}

The idea of $M_{3}$-rigidity evolves from Dehn's proof that the bar framework on the graph of a convex triangulated 3-polytope is infinitesimally rigid in 3-space [4]. Consider a convex triangulated 3-polytope in 3-space with $v$ vertices and $e=3 v-6$ edges. Its rigidity matrix $R$ is an $e \times v$ matrix whose columns are indexed by its vertices and rows by its edges and whose entries are in $\mathbb{R}^{3}$. In the row corresponding to the edge $\{x, y\}$, there are only two nonzero entries, namely $\mathbf{x}-\mathbf{y}$ in column $x$ and $\mathbf{y}-\mathbf{x}$ in column $y$. Take any face $a b c$. Let $R^{\prime}$ be the matrix obtained from $R$ by deleting the three rows corresponding to the three edges $a b, a c$, and $b c$, and the nine columns corresponding to the three vertices $a, b$, and $c$. Then the rank of $R$ is $3 v-6$ if and only if the rank of $R^{\prime}$ is $3 v-9$. Now $R^{\prime}$ is a $(3 v-9) \times(3 v-9)$ matrix. The nonzero terms of its determinant are products of $3 v-9$ terms comprising, for each $x \in V-\{a, b, c\}$, three distinct components corresponding to the three edges associated with it by the three matchings (see Fig. 7.1 for example).

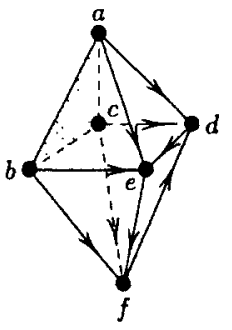

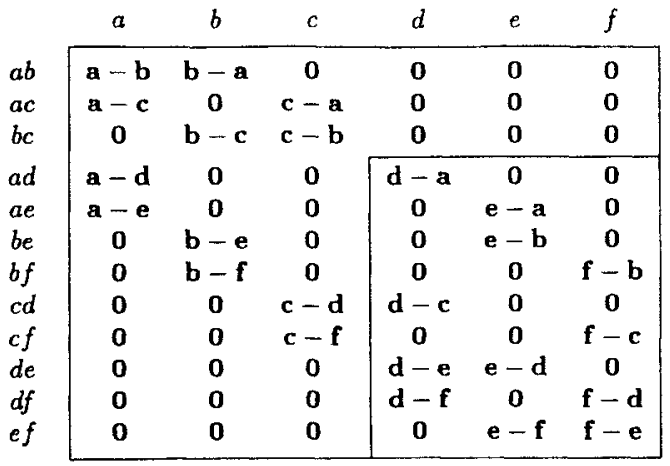

Fig. 7.1. The figure on the left shows the graph of an octahedron. The arrows show a set of three distinct matchings from edges to adjacent vertices (excluding the three vertices $a, b$, and $c$ ). There are two sets of such matchings. The matrix on the right shows the rigidity matrix of the octahedron. The submatrix enclosed in the smaller rectangle is the matrix $R^{\prime}$. det $R^{\prime}=[$ dace] [eabf] [ fbcd] + $[d a c f][e a b d][e a b d]$ [ fbce], where, for example, [dace] denotes the matrix whose rows are $\mathbf{d}-\mathbf{a}$, d - c, and d - e. The first term corresponds to the set of three matchings shown, while the second term corresponds to the other set. It is not hard to see, by convexity, that the two terms are of the same sign. 


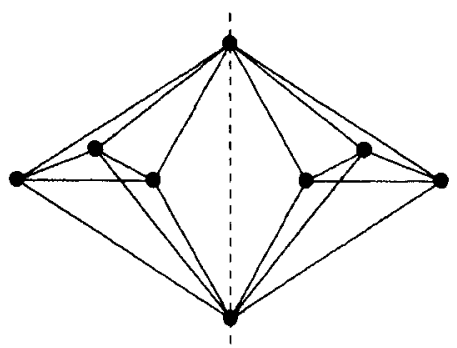

Fig. 7.2. A “2-bananas" graph.

Thus the truth of the condition $E^{*} \geq 3\left|V^{*}\right|$ for all $V^{*} \subseteq V-\{a, b, c\}$ is equivalent to the existence of a nonzero term in the Laplace expansion of det $R^{\prime}$, the determinant itself may be zero. The case where $\{a, b, c\}$ is not a face is similar but more subtle. $M_{3}$-rigidity of the polytope is equivalent to the existence of a nonzero term.

Dehn went further to show that all the terms in det $R^{\prime}$ were of the same sign, thus completing the proof of his theorem. However, it is not clear how Dehn's proof can be extended to arbitrary 2-manifolds.

We wish to emphasize here that $M_{3}$-rigidity is strictly weaker than generic 3-rigidity. An example is the "2-bananas" graph shown in Fig. 7.2. This graph is clearly not generically 3-rigid because in any realization in 3-space it can always rotate about the "hinge" indicated by the broken line. However, it is $M_{3}$-rigid by Proposition 4.3 with $t=6$.

Another difference is that while generically 3-rigid graphs are necessarily 3-connected in the vertex sense, $M_{3}$-rigid graphs may not be connected. Take an $M_{3}$-rigid graph $G$ with $v$ vertices and $e=3 v$ edges. (The 1-skeleton of any triangulated torus is an example of such a graph.) Then the graph obtained by taking the disjoint union of two copies of $G$ is disconnected and $M_{3}$-rigid by Proposition 4.3 with $t=0$.

\section{References}

1. D. Barnette: The minimum number of vertices of a simple polytope, Israel J. Math. 10 (1971), 121-125.

2. D. Barnette: Graph theorems for manifolds, Israel J. Math. 16 (1973), 62-72.

3. A. Björner: The minimum number of faces of a simple polytope, European J. Combin. 1 (1980), 27-31.

4. M. Dehn: Uber die Starrheit Knovexer Polyeder, Math. Ann. 77 (1916), 466-473.

5. A. Fogelsanger: The generic rigidity of minimal cycles, Ph.D. thesis, Cornell University, 1988.

6. M. Goresky and R. MacPherson: Intersection homology theory, Topology 19 (1980), 135-162.

7. M. Gromov: Partial Differential Relations, Springer-Verlag, Berlin, 1986.

8. G. Kalai: Rigidity and the lower bound theorem, I, Invent. Math. 88 (1987), 125-151.

9. Klee, V.: A $d$-pseudomanifold with $f_{0}$ vertices has at least $d f_{0}-(d-1)(d+2) d$-simplices, Houston J. Math. 1 (1975), 81-96.

10. J. R. Munkres: Elements of Algebraic Topology, Addison-Wesley, 1984.

11. R. P. Stanley: Interactions Between Commutative Algebra and Combinatorics, Birkhauser, Boston, 1983. 
12. T. S. Tay, N. L. White, and W. Whiteley: Skeletal rigidity of simplicial complexes, Report No. 20 , Institut Mittag-Leffler, 1992.

13. A. T. White: Graphs, Groups and Surfaces, North-Holland, Amsterdam, 1984.

14. W. Whiteley: Matroids and rigid structures, in N. White (ed.), Matroid Applications, Cambridge University Press, Cambridge, 1992, pp. 1-53.

Received May 13, 1993, and in revised form June 8, 1994. 